\title{
Relación entre la frecuencia de consumo de frutas y verduras, actividad física con el nivel de estrés académico en estudiantes del Instituto Tecnológico Superior Vida Nueva, Quito- Ecuador, 2016.
}

\section{Relationship between the frequency of consumption of fruits and vegetables, physical activity and the level of academic stress in students of Instituto Tecnológico Superior Vida Nueva, Quito, Ecuador, 2016.}

\author{
Pallo Oña Carmen Amelia; Mery Rodríguez Vásquez
}

Instituto Adventista Ecuador; EP Nutrición Humana, Universidad Peruana Unión

\begin{abstract}
Resumen
Objetivo: Determinar la relación entre la frecuencia de consumo de frutas y verduras y la actividad física con el estrés académico en estudiantes del Instituto Tecnológico Superior Vida Nueva, Quito, Ecuador, 2016. Materiales y métodos: enfoque cuantitativo, de tipo descriptivo, correlacional y de corte trasversal. La muestra estuvo conformada por 265 estudiantes de 1 ro, 2do y 3ro año. Para recolectar los datos se visitaron las aulas de los estudiantes. Se usó como herramienta el cuestionario de frecuencia de consumo de frutas y verduras, para evaluar la actividad física, se utilizó el cuestionario internacional de actividad física IPAQ y el inventario SISCO para medir el nivel de estrés. Resultados: se encontró que sólo el $1.1 \%$ de los estudiantes consumía todos los días frutas y $1.9 \%$ verduras. Además, se evidenció que el $37 \%$ de los estudiantes realizaron actividad física moderada, el $24.9 \%$ actividad física vigorosa y el $38.1 \%$ entre leve e inactivo. Por otra parte, el $76.7 \%$ presenta nivel de estrés académico moderado y el $22.5 \%$ niveles altos. Conclusión: Existe relación significativa entre el consumo de frutas y estrés académico ( $\mathrm{p}=.008$ ) mas no así, entre estrés académico y frecuencia de consumo de verduras, y actividad física ( $\mathrm{p}>5$ ).
\end{abstract}

Palabras claves: Consumo de frutas y verduras. Actividad física. Estrés académico

\begin{abstract}
Objetive. The main of the study was to determine the relationship between the frequency of consumption of fruits and vegetables and physical activity, with academic stress in students of the Superior Vida Nueva technologic institute, Quito, Ecuador, 2016. Materials and methods approach, descriptive - correlational type and transversal cutting. The sample was conformed by 265 students of 1st, 2nd and 3rd year. To collect the data, the classrooms of the students were visited. The tool was the frequency questionnaire of consumption of fruits and vegetables, to assess physical activity, the international questionnaire of physical activity IPAQ was used, and the SISCO inventory to measure the level of stress. According to Results, it was found that only the $1.1 \%$ of the students consumed fruit all the days and $1.9 \%$ vegetables. In addition, it was found that $37 \%$ of students performed a moderate physical activity, while the $24.9 \%$ vigorous physical activity and the $38.1 \%$ between mild and inactive. On the other hand, it was found that the $76.7 \%$ present a moderate academic level of stress and $22.5 \%$ high levels. As a conclusion, there is a significant relationship between the consumption of fruits and academic stress $(p=.008)$ but there is not a relationship between academic stress and frequency of consumption of vegetables, andphysical activity $(\mathrm{p}>5)$.
\end{abstract}

Keywords: consumption of fruits and vegetables. Physical activity. Academic stress.

\section{Introducción}

El ser humano consume alimentos para realizar actividades de forma regular, los vegetales, hortalizas, frutas, existen desde principio de las épocas antiguas, ya que estos tienen beneficios que han sido adjudicados por tener vitaminas, minerales y fibra, que ayudan al organismo a estar saludable evitando distintos tipos de infecciones y enfermedades tales como la diabetes, cáncer, enfermedades cardiovascular y otros (Araneda , 2015).

Por otro lado, la falta de actividad física ha ocasionado el 6\% de las muertes globales y ha sido catalogada como el cuarto factor de mortalidad, que ocasiona distintos tipos de cáncer y diabetes. Sin embargo el realizar actividad física moderada 150 minutos por semana y 30 minutos diarios trae beneficios para la salud, como efectos fisiológicos, que constituyen un factor protector de enfermedades crónicas no transmisibles; psicológicos que controlan la sensación de estrés académico y trastornos mentales que reducen la ansiedad, mejorando el estado de ánimo y el bienestar, aumentando la sensación de eficacia en el manejo de situaciones cotidianas (Barberan et al 2016 Lostaunau,2015 y Quispe et al 2016). 
Las relaciones que existen entre el estrés y la mala alimentación se vienen estudiando hace mucho tiempo, este es un trastorno que afecta a la población mundial, debido a que tienden a ingerir alimentos con alto contenido en grasas, azúcares, sal y calorías, el consumo en exceso de estos mismos, puede llevar no solo al sobrepeso y obesidad, sino también a aumentar el riesgo de desarrollar diversas patologías (Barattucci Y, 2011).

El consumo adecuado de frutas y verduras aporta vitaminas, minerales, antioxidantes que cumplen toda una serie de funciones en el organismo y previene enfermedades o infecciones. Por otro lado, el estrés está estrechamente relacionado con una mala alimentación y no sólo puede afectar la salud, sino también alterar el estado de ánimo y comportamiento. En relación a la actividad física, se conoce que realizar regularmente mejora la capacidad psicosocial, el estado de salud y disminuye el estrés físico y emocional (Nasser, 2013 ; FAO, 2003).

Existe evidencia sustancial de que el estrés puede afectar, no solo a través de procesos fisiológicos directos, sino también por el cambio de comportamientos que afecta la salud. Uno de estos comportamientos de salud es la dieta. Se ha demostrado que el estrés afecta la cantidad de alimento consumido. Algunos estudios Han demostrado que los individuos tienden ha aumentar el consumo de bocadillos altos en calorías y alto contenido de grasa cuando se sienten estresados, mientras que otros informaron que las personas comían menos de todos los alimentos cuando estaban bajo estrés. El estrés también se ha asociado con la selección de alimentos consumidos( Papier et al 2015).

El propósito de esta investigación es determinar la relación entre la frecuencia de consumo de frutas y verduras, actividad física con el nivel de estrés, para conocer y evitar que las consecuencias de la mala alimentación y el sedentarismo pueden afectar a la salud física y mental de los estudiantes. Así también fortalecer el conocimiento local y la importancia del trabajo profesional de nutrición, como parte de desarrollo y crecimiento a través de la promoción de hábitos basados en una alimentación y nutrición saludable, con el fin de mejorar el estado nutricional de la población joven y adulta.

\section{Metodología}

Este estudio fue de diseño no experimental de corte trasversal, de tipo descriptivo y correlacional, El tipo de muestreo fue no probabilístico por conveniencia bajo criterio del investigador, se trabajó con una muestra de 265 estudiantes de 1er, 2do, 3ro año, (Hombres 135 y 130 mujeres) del Instituto Tecnológico Superior Vida Nueva, entre las edades comprendidas 18 a 25 años.

Para recolección de los datos se utilizó tres instrumentos, uno para evaluar la frecuencia consumo de frutas y verduras, este instrumento fue creado por el Instituto Nacional de Cancerología y adaptado para este estudio. Está compuesto en dos partes: la primera está compuesto por 19 ítems de frecuencia de consumo de frutas y la segunda parte de igual número de ítems para el consumo de verduras y para el evaluar el nivel de actividad física se usó el cuestionario internacional de Actividad Física (IPAQ) (Formato breve) que tiene 7 preguntas y presenta las siguientes categorías de diagnóstico: Inactivo, Leve, Moderado, Vigorosa. Asimismo, los ítems están agrupados en tres áreas: actividad intensa (entre 5 a 7 días), actividad moderada (entre 3 a 4 días), actividad leve (en promedio $10 \mathrm{~min} /$ día) e inactivo. Y para la evolución del nivel de estrés, se usó el inventario de estrés académico (SISCO) Barraza en 2007. Este instrumento está conformado por 32 ítems. Los datos obtenidos fueron ingresados en una hoja de cálculo de Excel en su versión 2013; además, se utilizó el programa SPSS en su versión 22 para analizar los datos del estudio, el mismo que facilitó el análisis de las variables de estudio. Para realizar el análisis descriptivo se utilizó tablas de frecuencia y porcentaje, y para realizar el análisis de correlacional se utilizó el estadístico del Chi Cuadrado Pearson.

\section{Resultados y discusión}

Según la tabla 1 se muestra que solo el $1.1 \%$ de los estudiantes consumen frutas todos los días a diferencia del $98.9 \%$ menos de lo recomendado, se evidencia que solo el $1.9 \%$ consumen verduras siete días a la semana y el $98.1 \%$ menos de ello.

Tabla 1

Distribución de la frecuencia de consumo de frutas y verduras semanal

\begin{tabular}{lll}
\hline Consumo de frutas y verduras & $\mathrm{n}$ & $\%$ \\
\hline Frutas & & \\
Inadecuado $)(<7 \mathrm{v} / \mathrm{s})$ & 262 & 98.9 \\
Adecuado $(7 \mathrm{v} / \mathrm{s})$ & 3 & 1.1 \\
Verduras & & \\
Inadecuado $(<7 \mathrm{v} / \mathrm{s})$ & 260 & 98.1 \\
Adecuado $(7 \mathrm{v} / \mathrm{s})$ & 5 & 1.9 \\
Total & 265 & 100.0 \\
\hline
\end{tabular}


Según la tabla 2 se observa que el $61.9 \%$ de los jóvenes realiza actividad física entre moderada y vigorosa. Sin embargo, el $38.1 \%$ presentan niveles de actividad fisca entre inactivo y bajo.

Tabla 2

Distribución de la muestra según el nivel de actividad física

\begin{tabular}{lcc}
\hline Actividad física & $\mathrm{n}$ & $\%$ \\
\hline Inactivo & 36 & 13.6 \\
Bajo & 65 & 24.5 \\
Moderado & 98 & 37.0 \\
Vigorosa & 66 & 24.9 \\
Total & 265 & 100.0 \\
\hline
\end{tabular}

La tabla 3 muestra que el $74.7 \%$ de los estudiantes presenta un nivel de estrés académico moderado, mientras que el 21,9\% presenta niveles altos y solo $3.4 \%$ tienen estrés leve o sin estrés.

Tabla 3

Distribución de la muestra según el nivel de estrés académico

\begin{tabular}{|c|c|c|}
\hline Categoría de estrés & $\mathrm{n}$ & $\%$ \\
\hline Sin estrés & 7 & 2.6 \\
\hline Leve & 2 & 0.8 \\
\hline Moderado & 198 & 74.7 \\
\hline Alto & 58 & 21.9 \\
\hline Total & 265 & 100.0 \\
\hline
\end{tabular}

En la tabla4 se observa que existe relación estadísticamente significativa ( $\mathrm{p}>0.05)$ entre la frecuencia de consumo de fruta y el nivel de estrés en los estudiantes, mas no con la frecuencia de consumo de verduras. Por otro lado, el $74.3 \%$ de los estudiantes tienen estrés moderado consumen menos fruta y verduras.

Tabla 4

Análisis de la relación entre el consumo de consumo de frutas y verduras con el nivel de estrés.

\begin{tabular}{|c|c|c|c|c|c|c|}
\hline Frecuencia de consumo & $\begin{array}{l}\text { Estrés } \\
\text { Sin estrés }\end{array}$ & Leve & Moderado & Alto & Total & $\begin{array}{l}\text { Chi } \\
\text { cuadrado }\end{array}$ \\
\hline \multicolumn{7}{|l|}{ Frutas } \\
\hline $\operatorname{Inadecuado}(<7 \mathrm{v} / \mathrm{s})$ & $6(2.3 \%)$ & $2(0.8 \%)$ & $197(74.3 \%)$ & $57(21.5 \%)$ & $262(98.9)$ & \multirow[b]{2}{*}{$\begin{array}{l}X^{2}=.11 .72 \\
\mathrm{p}=.008\end{array}$} \\
\hline Adecuado (7v/s) & $1(0.4 \%)$ & $0(0 \%)$ & $1 \quad(0.4 \%)$ & $1(0.4 \%)$ & $3(1.1 \%)$ & \\
\hline \multicolumn{7}{|l|}{ Verduras } \\
\hline Inadecuado $(<7 \mathrm{v} / \mathrm{s})$ & $6(2.3 \%)$ & $2(0.8 \%)$ & $196(74.0 \%)$ & $56(21.1 \%)$ & $260(98.1 \%)$ & \multirow{3}{*}{$\begin{aligned} X^{2} & =.7 .43 \\
\mathrm{p} & =.059\end{aligned}$} \\
\hline Adecuado $(7 \mathrm{v} / \mathrm{s})$ & $1(0.4 \%)$ & $0(0 \%)$ & $2 \quad(0.8 \%)$ & $2 \quad(0.8 \%)$ & $5 \quad(1.9 \%)$ & \\
\hline Total & $7(2.6 \%)$ & $2(0.8 \%)$ & $198(74.7 \%)$ & $58(21.9 \%)$ & $265(100.0 \%)$ & \\
\hline
\end{tabular}

En la tabla 5 demuestra que no existe relación estadísticamente significativa entre la actividad física con el nivel de estrés. Se observó que los estudiantes que realizan actividad física vigorosa y moderada tienen un nivel de estrés moderado y alto, los que tuvieron actividad física leve o sin estrés son los que realizan menos actividad física. 
Tabla 5

Análisis de la relación entre actividad física y estrés académico.

\begin{tabular}{|c|c|c|c|c|c|c|}
\hline \multirow[b]{2}{*}{$\begin{array}{l}\text { Actividad } \\
\text { física }\end{array}$} & \multicolumn{4}{|c|}{ Estrés } & \multirow[b]{2}{*}{ Total } & \multirow[b]{2}{*}{ chi cuadrado } \\
\hline & Sin estrés & Leve & Moderado & Alto & & \\
\hline Sedentario & $0(0.0 \%)$ & $0(0.0 \%)$ & $26(9.8 \%)$ & $10(3.8 \%)$ & $36(13.6 \%)$ & \multirow{5}{*}{$\begin{aligned} X^{2} & =0.576 \\
\mathrm{p} & =.763\end{aligned}$} \\
\hline Bajo & $2(0.8 \%)$ & $1(0.4)$ & $51(19.2 \%)$ & $11(4.2 \%)$ & $65(24.5 \%)$ & \\
\hline Moderado & $4(1.5 \%)$ & $1(0.4 \%)$ & $69(26.0 \%)$ & $24(9.1 \%)$ & $98(37.0 \%)$ & \\
\hline Vigoroso & $1(0.4 \%)$ & $0(0.0 \%)$ & $52(19.6 \%)$ & $13(4.9 \%)$ & $66(24.9 \%)$ & \\
\hline Total & $7(2.6 \%)$ & $2(0.8 \%)$ & $198(74.7 \%)$ & $58(21.9 \%)$ & $265(100.0 \%)$ & \\
\hline
\end{tabular}

\section{Discusión}

Las frutas y verduras constituyen uno de los elementos esenciales de una alimentación saludable, por su alto contenido de vitaminas, minerales, fibras y antioxidantes, juegan un papel importante en la prevención y tratamiento de diversas enfermedades crónicas no transmisibles, entre otras Macui et al. (2015). Por otro lado, la actividad física está relacionada con los niveles de estrés, dependiendo de su intensidad, es decir a mayor esfuerzo físico menos estrés percibido (Norris, 1992). El estrés está estrechamente relacionado con una mala alimentación y no solo puede afectar la salud, sino también alterar el estado de ánimo y comportamiento. Por otro lado, en relación a la actividad física, se conoce que realizar regularmente mejora la capacidad psicosocial, el estado de salud y disminuye el estrés físico y emocional (Nasser, 2013; Lostaunau, 2012).

Al evaluar la frecuencia de consumo de frutas, se evidenció adecuada ingesta $1.1 \%$ para frutas y $1.9 \%$ para verduras. Asimismo, Alsunni et al. (2015), encontraron que el $84.4 \%$ de los estudiantes no consumían lo recomendado según OMS de 3 a 5 porciones, estos resultados coinciden con los mencionados en otros estudios Tassitano et al. (2014), quienes evidenciaron que la mayor proporción de la muestra $65.8 \%$ tenía un insuficiente consumo de frutas y verduras. Sin embargo, En el estudio de Sánchez et al. (2015), encontraron que menos de la mitad de su población tuvo una baja ingesta de verduras 39.8\% más no en frutas $73.9 \%$. Este estudio evidencio que la alteración del patrón alimentico corresponden a los cambios ocurridos en los últimos años en los países industrializados donde el consumo de alimentos proteicos o expensas sobre todo las carnes rojas han aumentado, generando disminución de la ingesta de leguminosas, frutas y verduras.

Estudios muestran que existen factores que probablemente influyen en la decisión de consumo de frutas y verduras, estos factores son culturales, económicos y cognitivos. Algunos de los estudiantes necesitan trabajar para costear sus estudios en la institución privada, por lo que destinan menos dinero para comprar alimentos como frutas y verduras. También algunos de ellos traen consigo hábitos de consumo de alimentos malsanos, aprendidos en el hogar. Por último, existe escaso conocimiento sobre los beneficios que aporta consumir la cantidad y frecuencia adecuada de frutas y verduras.

Respecto a la actividad física, se observó que el 37\% tienen nivel moderado y el $24.9 \%$ actividad vigorosa. Estos resultados coinciden con los mencionados por Barberan (2016), quienes encontraron que el $42.7 \%$ de los encuestados tuvieron un nivel de actividad física moderada y el $25.8 \%$ presenta nivel alto, probablemente esto se debe a la etapa universitaria que suele presentar altas cargas académicas, por lo que realizar actividad física podría reducir el riesgo de padecer estrés generado por el cumplimiento de tareas. No obstante, un estudio liderado por Arias et al (2014)encontraron que más de mitad de los evaluados (54.5\%) tuvieron actividad física moderada y mencionan que los estudiantes necesitan realizar actividad física para mantenerse sanos y con energía durante el día. Sin embargo Rubio et al. (2016), encontraron que la mayoría de los estudiantes realizaban actividad física moderada y alta (77\%), esto probablemente se deba al sesgo que se tiene de una pregunta formulada para recordar que actividad física se realiza en los últimos 7 días, lo que conlleva a olvidar y no responder correctamente. Hay que mencionar, que el mismo estudio refiere que lo hacen por motivos de salud e imagen personal, sobre todo en las mujeres.

Al evaluar el nivel de estrés académico, los resultados mostraron que el 74.7\% de los estudiantes tuvieron estrés moderado y estrés alto un $21.9 \%$. Córdova et al. (2015), encontraron que el $83.3 \%$ de los estudiantes tuvieron estrés moderados. No obstante, Ornelas (2016), qué menos de la mitad tuvieron estrés moderado 48.9\%. Sin embargo, Versaevel ( 2015), encontró que el 34.5\% de los estudiantes presentaron nivel de estrés académico moderado. Probablemente eso se deba al cambio de ambiente del estudiante, el trabajo, el estudio y la responsabilidad que adquiere para forjar su futuro. Los estresores más predominantes fueron el tipo de trabajos que mandan los profesores (33.2\%); además, el $60.6 \%$ de los estudiantes rara vez tienen habilidad asertiva para defender sus creencias, preferencia de ideas pensamientos sin dañar a otros también se encontró que el 69.4\% rara vez o nunca han elaboración un plan o ejecución de tareas y finalmente el 67.2\% algunas veces se elogió a sí mismo. De la misma manera, Cárdenas et al. (2014), encontraron en su estudio que la falta de tiempo para cumplir con las tareas académicas es uno de los factores que influyen en el estrés y por otro lado, las estrategias de afrontamiento que utilizaron los estudiantes fue la elaboración de un plan de ejecución de 
sus tareas y búsqueda de información sobre la misma. No obstante, Ortiz et al. (2012) encontraron que la estrategia de afrontamiento que utilizaron más fue generar estrategias cognitivas y conductuales encaminadas a solucionar los problemas.

Con respecto al análisis de correlación entre la frecuencia de consumo de frutas y el estrés académico, se evidenció que existe relación estadísticamente significativa ( $\mathrm{p}<.008)$, teniendo en cuenta que el 95\% de los estudiantes tiene estrés entre moderado y alto, solo el $1.1 \%$ consume frutas a diferencia de las verduras, donde no se encontró correlación ( $p>0.05$ ). Estudios como el de Mikolajczyk et al. (2009) encontraron asociación significativa $(<0.001)$ entre el bajo consumo de frutas y verduras con estrés y depresión en estudiantes mujeres que tenía un consumo alto de alimentos procesados, por lo que se evidencia que existe alimentos estimulantes y excitantes que alteran el sistema nervioso central, logrando cambios en la conducta alimentaria. Por lo que una alimentación rica en nutrientes vivos como frutas y verduras que contiene vitamina A, C, E y minerales como magnesio potasio, disminuye la tensión y sus efectos del estrés. No obstante Fabián et al. (2013), al evaluar el consumo de frutas y verduras e ingresos mensuales, IMC y estrés académico no encontraron mayor significancia, esto probablemente porque sus padres tienen ingresos bajos que no permiten conseguir frutas y verduras en cualquier momento. Además, los factores de estrés académico conducen al desarrollo de nuevas tendencias o patrones alimentarios, muchas veces debido a la falta de tiempo, nuevos tipos de alimentos procesados, horarios y la distancia entre el lugar de estudio y la residencia del estudiante.

Referente a la actividad física y su relación con el estrés académico, no se evidenció correlación estadísticamente significativa entre las variables ( $\mathrm{p}>0.05$ ). Posiblemente se deba que los estudiantes no recordaban las actividades específicas de los últimos 7 días que recomienda la OMS o no respondieron con sinceridad a las preguntas formuladas. Sin embargo, Lostaunau. (2015) encontró correlación estadísticamente significa entre actividad física y estrés académico ( $\mathrm{p}>0.033$ ). De igual manera, en un estudio comparativo realizado por Steptoe et al. (1996) en un grupo de estudiantes universitarios, observaron que la actividad física disminuye el estrés académico. Estas investigaciones se asemejan con los resultados encontrados Kubota et al.(2006) donde encontraron relación significativa $(\mathrm{p}<001)$ en los estudiantes del grupo de actividad física de intensidad moderada y vigorosa que mostraron una menor tendencia a expresar estrés, reducir el riesgo de desarrollar enfermedades psiquiátricas relacionadas, como la depresión. Asimismo, Al-Eisa et al. (2014), en un estudio en estudiantes saudíes, evidenciaron que a mayores niveles de actividad física existe mejor salud mental de la población de estudio.

\section{Conclusiones}

Finalizado el trabajo se puede concluir que existe relación significativa entre el consumo de frutas y estrés académico $(\mathrm{p}=.008)$ mas no así, entre estrés académico y frecuencia de consumo de verduras, y actividad física $(\mathrm{p}>5)$.

\section{Referencias}

Al-Eisa, E., Buragadda, S., \& Melam, G. (2014). Association between physical activity and psychological status among Saudi female students. BMC Psychiatry, 14(1), 238.

Alsunni, A., \& Ahmed, B. (2015). Fruit and vegetable consumption and its determinants among Saudi university students. Journal of Taibah University Medical Sciences, 10(2), 201-207.

Araneda, J., Ruiz, M., Vallejos, T., \& Oliva, P. (2015). Consumo de frutas y verduras por escolares adolescentes de la ciudad de Chillán . Revista Chilena Nutricion.

Arias, K., Garrindo, A., Guerrero, J., \& Bermudez, M. (2014). Prevalencia de Actividad Física en los Estudiantes de la Universidad del Magdalena, Santa Marta, Colombia, 2012. Salud Publica, 3(422), 15-22.

Barattucci Y. (2011). Estrés y Alimentación Universidad Fasta Facultad de Ciencias en nutricion., 1-81.

Barberan, J., \& Trejos, J. (2016). Actividad Fisica en Universitarios y su relacion con Aueficacia y persepsion de riesgo. Pontifica Universidad Javeriana.

Fabián, C., Ideliz, P., Ríos, J., Betancourt, J., Cruz, S., González, A., ... Rivera, W. (2013). Dietary Patterns and their Association with Socio- demographic Characteristics and Perceived Academic Stress of College Students in Puerto Rico. Programa de Nutrición, Departamento de Desarrollo Humano, Escuela de Posgrado de Salud Pública, 32(1), 36-43.

FAO. (2003). Prioridad mundial al consumo de fruta y hortalizas. Retrieved from

Kubota, T., Ohmori, H., \& Munacata, T. (2006). The Relationship between Physical Activity Level and Stress Response in University Students.

Lostaunau, P. (2015). Indice de masa corporal, consumo de alimentos y actividad física en relación con el estrés en adolescentes de una Institución Educativa Privada, Villa María del Triunfo-2014. Universidad Nacional San Marcos.

Lostaunau, P., García, R., Pérez, F., Pérez, J., \& Natividad, L. (2012). Evaluación del estrés académico en estudiantes de nueva incorporación a la universidad. Revista Latinoamericana de Psicología. Universidad Nacional San Marcos. Retrieved from http://openjournal.konradlorenz.edu.co/index.php/rlpsi/article/view/1038

Macui, C., \& Ponce, J. (2015). Frecuencia de consumo de verduras y frutas y su relación con el estreñimiento en estudiantes de la Carrera de Terapia Física que cursan del primer al tercer semestre de la Facultad de Ciencias Médicas. Universidad Catolica de Santiago de Guayaquil. 
Mikolajczyk, R., El Ansari, W., \& Maxwell, A. (2009). Food consumption frequency and perceived stress and depressive symptoms among students in three European countries. Nutrition Journal, 8(1), 31.

Nasser, H. (2013). Stress , Lifestyle, and Diet in College Students : Analysis of the YEAH Study. Digital Commons. University of Rhode Island.

Norris, R., Carroll, D., \& Cochrane, R. (1992). The effects of physical activity and exercise training on psychological stress and well-being in an adolescent population. Journal of Psychosomatic Research, 36(1), 55-65.

Ornelas, M. (2016). Evaluacion del estres academico. Revista Educarnos. Retrieved from

Papier, K., Ahmed, F., Lee, P., \& Wiseman, J. (2015). Stress and dietary behaviour among first-year university students in Australia: Sex differences. Nutrition, 31(2), 324-330.

Quispe, J. (2016). Nivel de Actividad Fisica en Adolescentes de un distrito de la region Callao., 33(2), 1-8.

Rubio, R., \& Varela, M. (2016). Barreras percibidas en jóvenes universitarios para realizar actividad física. Perceived Barriers in University Students to Physical Activity Practice., 42(1), 61-69.

Sánchez, V., \& Aguilar, A. (2015). Hábitos alimentarios y conductas relacionadas con la salud en una población universitaria. Nutricion Hospitalaria, 31(1), 449-457.

Tassitano, R., Tenorio, M., Cabral, P., \& Pontes Da Silva, G. (2014). Clustering of physical inactivity and low fruit and vegetables intake and associated factors in young adults Agregamento entre a inatividade física e consumo de frutas, legumes e verduras e fatores associados em adultos jovens. Brazilian Journal of Nutrition, 27(1), 25-34.

Versaevel, L. (2015). Canadian Post-Secondary Students, Stress, and Academic Performance - A Socio-Ecological Approach. Western University. 